\title{
Per-oral endoscopic myotomy for palliation of achalasia: A video atlas
}

Siva Raja, MD, PhD, FACS, ${ }^{\mathrm{a}}$ Saurav Adhikari, MD, ${ }^{\mathrm{a}}$ and Madhu R. Sanaka, MD, ${ }^{\mathrm{b}}$ Cleveland, Ohio

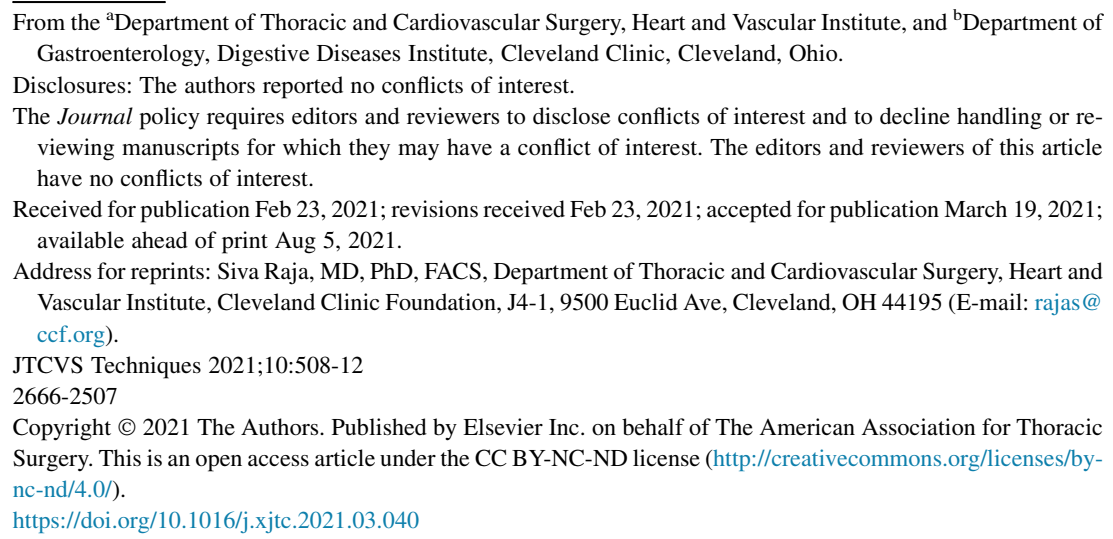

$\checkmark$ Video clip is available online.

Feature Editor's Introduction-The crystallization of a procedure into a series of sequential steps may be the most basic, yet most powerful, framework for understanding, and teaching, an operation. The late 94th President of the American Association for Thoracic Surgery, David J. Sugarbaker, is oft-regarded as a master educator and visionary in our field. His residents and fellows will fondly remember their requirement to write the 10 steps of every operation on the white board in the operating room, before every case. They may even submit that this exercise establishes a cognitive framework that optimizes procedural learning through planning, expectation, encounter, and result. Video Atlas Articles at the Journal of Thoracic and Cardiovascular Surgery Techniques are purposed to stimulate this cognitive framework of surgical education in an enduring and peer-reviewed format. In the Video Atlas Article that follows, one of the most complex endoscopic procedures, and one that has gained remarkable traction over the past decade, per-oral endoscopic myotomy, is expertly distilled into a series of 9 successive steps, with each step taught through its own narrated video and corresponding short text description.

\section{Bryan M. Burt, MD}

Achalasia is a rare esophageal motility disorder with surprisingly numerous therapeutic options. Historically

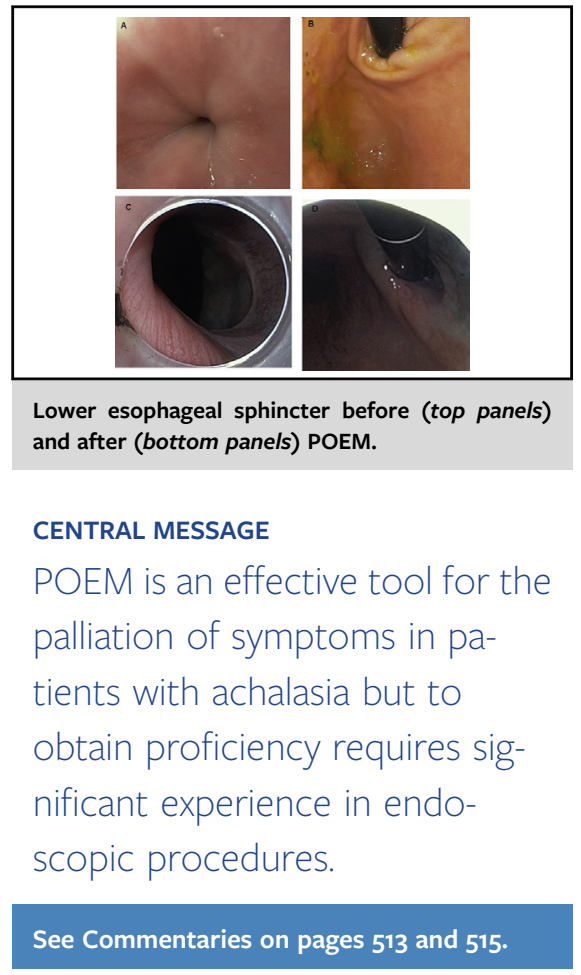

morbid options such as a thoracic Heller myotomy are now performed for the palliation of symptoms via minimally invasive approaches with minimal morbidity and quick return to function. Similarly, imprecise endoscopic therapies such as pneumatic balloon dilation are now being replaced with endoscopic myotomy known as POEM (per oral endoscopic myotomy). While both Heller myotomy and POEM have their staunch supporters, they likely have their specific indications with some overlap.

We have previously published our algorithm for the treatment of achalasia, where the role of POEM is prominent. ${ }^{1}$ POEM is the treatment of choice for patients with type III achalasia, previous myotomy, severely dilated esophagus, and in the frail patient. POEM is reasonable in patients with type II achalasia and in those without a dilated esophagus, but given the increased risk of gastroesophageal reflux disease (GERD), it has not been our group's first choice.

\section{RESULTS}

\section{Steps for Performing a POEM}

For instructional purposes, the videos may be a composite of different cases. 
1. Placement of an over-the-scope tube and dissection cap, and examination of the lower esophageal sphincter (LES; Video 1). An over-the-scope tube is placed in the proximal esophagus to decrease trauma from repeated exchange of the endoscope. The dissection cap is a key tool in the tunneling process. It is important have a short footprint for the cap adjacent to the area where triangular tip (TT) cautery knife exits the scope to decrease thermal burn to the cap as well as to avoid accidental entrapment of the TT knife on the cap edge. Only $\mathrm{CO}_{2}$ insufflation should be used and air should be avoided.

2. Measurement of distance from incisors, possible lavage (no video). If food debris is noted in the esophagus, this should be thoroughly cleared. The measurement of the LES as a distance from the incisors is an important step. However, relying this measurement alone can often lead to an incomplete myotomy. This phenomenon is noted in sigmoidal and/or dilated esophagus, as the distance traveled to the LES within the center of the lumen may be significantly shorter than the distance traveled within the wall of the esophagus. As a result, we recommend establishing the entry point for the submucosal tunnel by measuring the distance back from the LES along the proposed track of the tunnel. We generally use 9 to $12 \mathrm{~cm}$ from the LES with shorter tunnel for very sigmoidal esophagus.

3. Creation of submucosal bleb (Video 2). The location of the mucosal entry can be anterior or posterior based on the preference of the endoscopist. We prefer an anterior entry for primary POEM and a posterior entry for a redo POEM, given the likely anterior location of previous abdominal myotomy. We create a submucosal bleb by injecting a saline solution mixed with methylene blue and a diluted epinephrine solution $(1: 250,000)$. Some

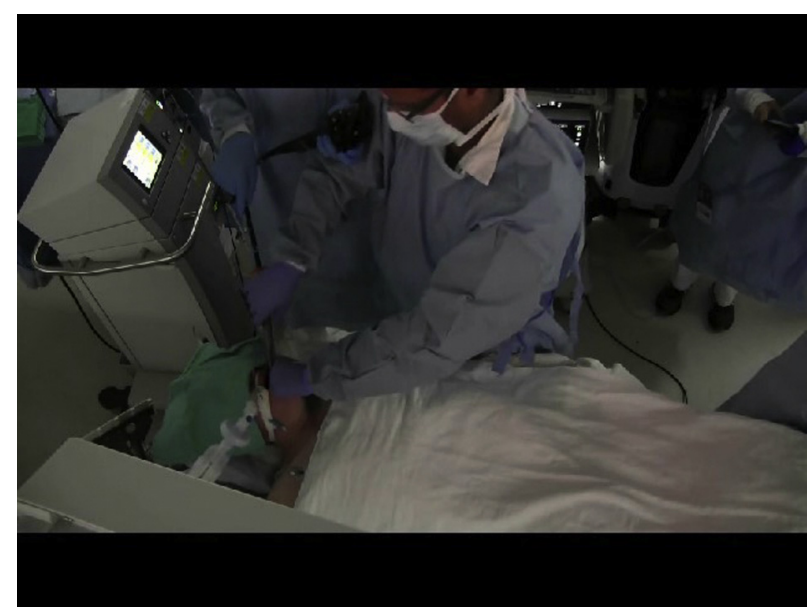

VIDEO 1. Video available at: https://www.jtcvs.org/article/S26662507(21)00513-7/fulltext.

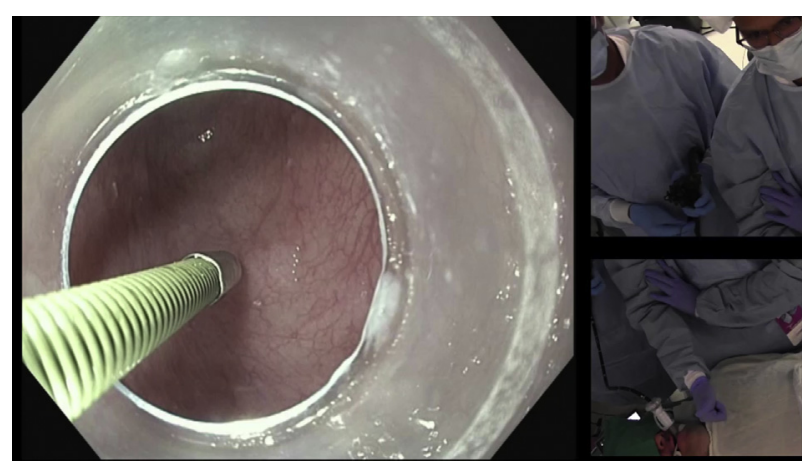

VIDEO 2. Video available at: https://www.jtcvs.org/article/S26662507(21)00513-7/fulltext.

practitioners avoid the use of epinephrine for a theoretic risk of flap necrosis, but we have not found this to be a concern in practice. Similarly, some practitioners use viscous solutions in the creation of the bleb to increase the time to deflation of the bleb. We had noticed increased bubbling from such solutions, which obscure our view limiting its use in our practice.

4. Mucosotomy and entry into the third space (Video 3). After the creation of the bleb, the TT knife is used to enter the space between the submucosa and the inner circular muscle (aka, third space). The mucosotomy is created for a length of about $1.5 \mathrm{~cm}$. It is important to avoid injury to the muscle layer, as it can lead to a delayed leak in the postoperative setting. In addition, subcutaneous emphysema may be noticed at this point, but if the muscle layer is intact, the procedure can still proceed without detriment to the patient.

5. Creation of a submucosal tunnel (Video 4). Once the mucosotomy is created, a tunnel is progressed to about 3 to $5 \mathrm{~cm}$ beyond the expected distance for the LES. Methylene blue solution is liberally used for both the purpose of hydrodissection as well as to improve visualization by increasing the contrast between tissues for a safer dissection. It is also important to frequently check the

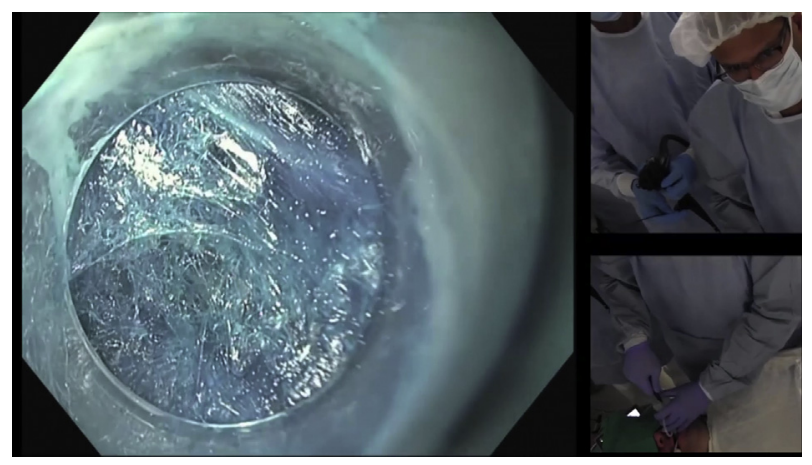

VIDEO 3. Video available at: https://www.jtcvs.org/article/S26662507(21)00513-7/fulltext.

JTCVS Techniques $・$ Volume 10, Number C 


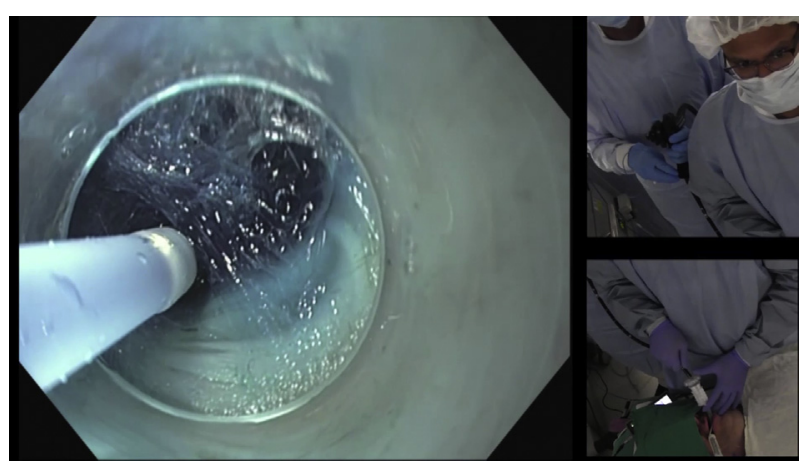

VIDEO 4. Video available at: https://www.jtcvs.org/article/S26662507(21)00513-7/fulltext.

progress of the tunnel within the lumen of the esophagus to avoid spiraling. Should there be bleeding noted, care should be taken in achieving hemostasis. We can use cautery easily for bleeding from the muscle side. For bleeding from the mucosal side, we use a coagulation grasper and a short energy burst to decrease thermal injury.

6. Inspection of the submucosal flap (Video 5). At the completion of the submucosal tunneling, one should check the integrity of the submucosal flap to rule out accidental perforation. In addition, one should also enter the stomach and view the LES via retroflexion to ensure adequate forward progress of the blue dye. It should be noted that the leading edge of the blue dye can be several centimeters beyond the end of the tunnel. In addition, minor thermal blistering from thermal transfer to the mucosa can be noted and do not generally result in delayed leaks.

7. Myotomy (Video 6). After the integrity of the mucosal flap is established, a myotomy is started 2 to $3 \mathrm{~cm}$ from the distal edge of the mucosotomy. It is important to have several centimeters where all layers are intact to cause the tunnel to seal. The area of greatest risk for injury is the LES, where there may be decreased room between the mucosal flap and the muscle layer. The

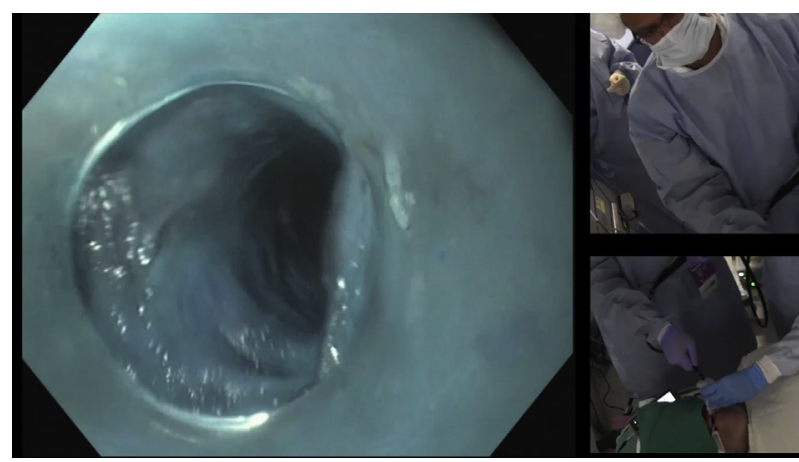

VIDEO 5. Video available at: https://www.jtcvs.org/article/S26662507(21)00513-7/fulltext.

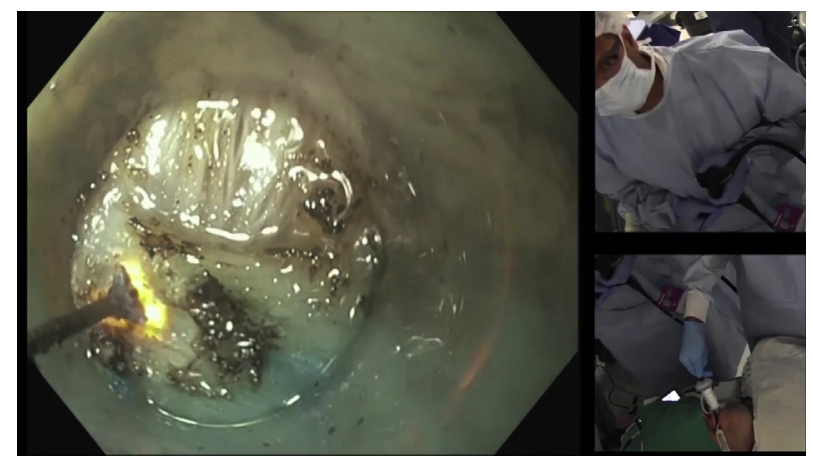

VIDEO 6. Video available at: https://www.jtcvs.org/article/S2666 2507(21)00513-7/fulltext.

myotomy is carried to the end of the tunnel. At the completion of this we confirm the adequacy of the myotomy by re-examining the LES, which should be patulous.

8. Mucosotomy closure (Video 7). The mucosotomy defect is closed in with endoscopic clips. It is important to adequately reapproximate and evert both edges and start from distal extent to the proximal extent. Should there be asymmetric clipping or bunching of the tissue flap on one side, it could lead to contrast pooling on postprocedure barium esophagogram. As such, the specter of contained perforation will be raised. In our practice, we have found this radiographic finding to not have a clinical consequence, allowing it to be managed conservatively.

9. Examination of upper esophageal sphincter. When removing the endoscope after decompressing the stomach, it is important to check the upper esophageal sphincter for potential damage from the over the scope tube.

\section{DISCUSSION}

Since the first reports by Inoue and colleagues, ${ }^{2}$ there has been a significant number of publications that have examined numerous permutations of this technique. There was

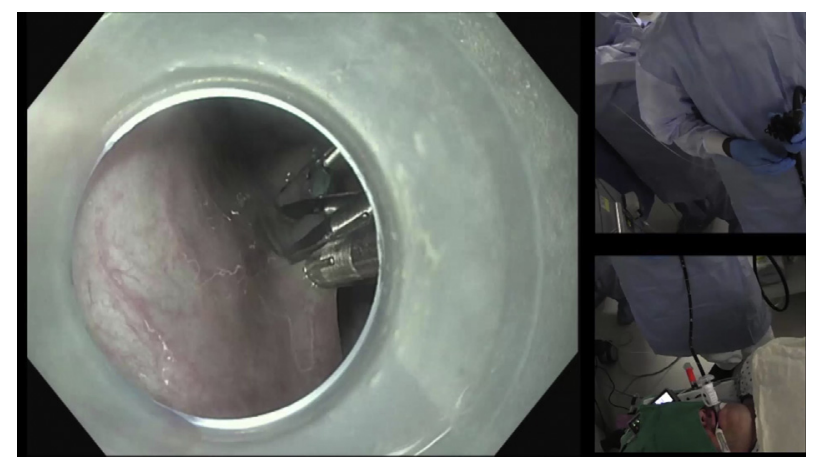

VIDEO 7. Video available at: https://www.jtcvs.org/article/S2666 2507(21)00513-7/fulltext. 
early enthusiasm for partial-thickness/circular myotomy with the idea that this might decrease reflux, but studies thus far have been inconclusive. ${ }^{3,4}$ The only obvious advantage is that a full-thickness myotomy is quicker to perform. In our experience, we perform a full-thickness myotomy, as we had a few recurrences early in our series where a circular myotomy was employed. Similarly, studies have also examined the performance of the myotomy on the anterior surface versus the posterior surface. This concept too has its staunch supporters for both camps. While a small series found increased GERD after a posterior myotomy, others have found no statistically significant difference in efficacy and postoperative GERD. ${ }^{5,6}$ We have found posterior myotomy more challenging from a visualization standpoint as the dissection fluid pools in the tunnel but its mucosotomy is easier to close. For the purposes of standardization in a large-volume practice, we perform the myotomy in patients with primary POEM anteriorly on the lesser curve, as our routine in Heller myotomies is to perform the myotomy anteriorly on the greater curve. This allows us to more easily salvage a potential failure of one technique with the other. We reserve the posterior myotomy for those who have had a previous anterior myotomy from a previous POEM or abdominal Heller.

The length of the myotomy is also commonly a subject of debate. The early myotomies had long esophageal myotomy. From several decades of experience with transabdominal Heller myotomies, we know that 4 to $5 \mathrm{~cm}$ of esophageal myotomy usually is adequate for long-term palliation. As such, most centers have now limited their esophageal myotomy to 5 to $7 \mathrm{~cm}$. The exception to this is in the setting of type III achalasia and in sigmoidal esophagus. A longer myotomy is reasonable in the treatment type III achalasia to address the spastic muscle tissue. Even here, we generally limit the myotomy to $10 \mathrm{~cm}$ or less. In sigmoidal or dilated esophagus, challenging angles can result in tearing the mucosal entry site. The substantially greater distance to the LES within the wall of the esophagus compared with the luminal measurement can also result in spiraling within the tunnel. As such, we recommend a short tunnel when the esophageal morphology is end stage. Given that the gastroesophageal junction is in plain sight during a Heller myotomy, traversing the gastroesophageal junction adequately is generally not a problem during that procedure. However, during a POEM, tunneling in the esophageal wall of a dilated esophagus can underestimate the distance traveled and result in an inadequate gastric myotomy. To avoid this, many techniques such as laparoscopic confirmation and fluoroscopy have been used. We choose to make a longer myotomy on the gastric side $(4-5 \mathrm{~cm})$ to compensate for any errors in measurement.

The technique we have developed is a 2-person POEM technique. ${ }^{7}$ This allows us to keep continuous forward pressure in the tunnel to facilitate quicker dissection as well as to maximize the distance of the mucosal flap from the muscle layer. We find most value in the 2-person technique in a redo field, where the precision offered by this technique helps avoid accidental mucosal perforation. Significant skill and experience in endoscopy are needed to perform POEM. As such, this also allows for better control when lessexperienced trainees are involved in the procedure, where that staff can better manipulate the field of dissection. In the most clinical settings, including in our own practice, it may not always be feasible to have 2 skilled practitioners, and the technique can easily be performed by a single experienced endoscopist.

Standard postoperative evaluation involves a barium swallow on the first postoperative day and a minimum of 1 month of anti-acid therapy with proton pump inhibitors and topical agents such as sucralfate. Failure to adequately suppress acid in the immediate postoperative period had led to ulcer formation and upper gastrointestinal bleeding in our early experience. At 2 months, patients undergo a timed barium esophagogram and a Bravo $\mathrm{pH}$ evaluation off medications. Postoperative GERD can be noted in up to $50 \%$ of patients. ${ }^{1,6,8}$ As such, objective evaluation for GERD is critical in the continued management of patients with achalasia.

\section{Risks and Limitations}

POEM is generally a safe operation with low morbidity and rare mortality. However, it requires advanced surgical and endoscopic skills and entails a steeper learning curve. Extreme sigmoidal morphology of the esophagus, severe dilation, scarring due to previous surgery, and submucosal fusion all present technical difficulties during POEM. The robust submucosal blood supply carries a potential bleeding risk. Both endoscopy and submucosal tunnel creation carry potential risk of mucosal perforation, which is usually managed by closure with clips. ${ }^{9}$ Excessive capnoperitoneum or capnothorax may also require decompression.

\section{CONCLUSIONS}

POEM is an effective tool for the palliation of symptoms in patients with achalasia. To become skilled in performing POEM, a robust endoscopic experience is a necessary prerequisite. The future of this procedure in the arsenal of the achalasia surgeons may be tempered by its increased risk of postoperative reflux.

\section{References}

1. Raja S, Murthy SC, Tang A, Siddiqui HU, Parikh MP, Ahmad U, et al. Per oral endoscopic myotomy: another tool in the toolbox. J Thorac Cardiovasc Surg. 2019;158:945-51.

2. Inoue H, Minami H, Kobayashi Y, Sato Y, Kaga M, Suzuki M, et al. Peroral endoscopic myotomy (POEM) for esophageal achalasia. Endoscopy. 2010;42:265-71.

3. Li QL, Chen WF, Zhou PH, Yao LQ, Xu MD, Hu JW, et al. Peroral endoscopic myotomy for the treatment of achalasia: a clinical comparative study of endoscopic full-thickness and circular muscle myotomy. J Am Coll Surg. 2013;217: $442-51$. 
4. Wang XH, Tan YY, Zhu HY, Li CJ, Liu DL. Full-thickness myotomy is associated with higher rate of postoperative gastroesophageal reflux disease. World J Gastroenterol. 2016;22:9419-26.

5. Ramchandani M, Nabi Z, Reddy DN, Talele R, Darisetty S, Kotla R, et al. Outcomes of anterior myotomy versus posterior myotomy during POEM: a randomized pilot study. Endosc Int Open. 2018;6:E190-8

6. Khashab MA, Sanaei O, Rivory J, Eleftheriadis N, Chiu PWY, Shiwaku H, et al. Peroral endoscopic myotomy: anterior versus posterior approach: a randomized single-blinded clinical trial. Gastrointest Endosc. 2020;91: 288-97.e7.
7. Sanaka MR, Jegadeesan R, Thota PN, Navaneethan U, Lopez R, Murthy SC, et al Two-person technique of peroral endoscopic myotomy for achalasia with an advanced endoscopist and a thoracic surgeon: initial experience. Can J Gastroenterol Hepatol. 2016;2016:2656101.

8. Schlottmann F, Luckett DJ, Fine J, Shaheen NJ, Patti MG. Laparoscopic Heller myotomy versus peroral endoscopic myotomy (POEM) for achalasia: a systematic review and meta-analysis. Ann Surg. 2018;267:451-60.

9. Haito-Chavez Y, Inoue H, Beard KW, Draganov PV, Ujiki M, Rahden BHA, et al Comprehensive analysis of adverse events associated with per oral endoscopic myotomy in 1826 patients: an international multicenter study. Am J Gastroenterol. 2017;112:1267-76. 